\title{
Measurement of local permeability at subcellular level in cell models of agonist- and ventilator-induced lung injury
}

\author{
Oleksii Dubrovskyi, Anna A Birukova and Konstantin G Birukov
}

\begin{abstract}
Alterations of cell monolayer integrity and increased vascular permeability are key to many pathologies, including atherosclerosis, stroke, lung injury, cancer, digestive disorders and others. Current approaches to probe cell permeability require specific culture conditions and provide an average estimation of trans-monolayer permeability, while analysis of regional monolayer permeability in static and mechanically challenged monolayer at a single-cell scale resolution remains unavailable. We describe a novel method for visualization and rapid quantification of trans-monolayer permeability based on high-affinity interactions between ligand (FITC-conjugated avidin) added in the culture medium, which permeates cell monolayer to reach substrate-bound acceptor (biotinylated gelatin or collagen). This approach was used to simultaneously evaluate general and local permeability responses by endothelial cell (EC) monolayer to a spectrum of barrier protective and barrier disruptive agonists and their combinations. The results revealed the paracellular pathway as the predominant mechanism of agonist-induced mass transport by pulmonary EC. We also detected for the first time, in a direct assay, a synergistic effect of pathologically relevant levels of cyclic stretch (CS) and edemagenic agent thrombin in the development of pulmonary EC hyper-permeability response observed in ventilator-induced lung injury. The reported novel assay provides unique information about local monolayer permeability changes induced by agonists, mechanical factors or molecular perturbations in single cells. However, the spectrum of substrates, assay formats and experimental conditions compatible with this assay suggest its broad application in the areas of endothelial and epithelial biology, cancer research and other fields.
\end{abstract}

Laboratory Investigation (2013) 93, 254-263; doi:10.1038/labinvest.2012.159; published online 19 November 2012

KEYWORDS: cytoskeleton; endothelial dysfunction; intercellular gaps; pathological mechanical strain; permeability; vascular leak; visualization

Changes in cell monolayer barrier integrity and compromised barrier function are key features of many pathological conditions, including atherosclerosis, ${ }^{1}$ cancer, ${ }^{2}$ stroke, ${ }^{3}$ inflammation, pulmonary edema ${ }^{4,5}$ and others. The methods for in vitro evaluation of mass transport across the cell monolayer have wide applications in studies addressing mechanisms of cell monolayer integrity, regulation of blood-gas, blood--brain and other barriers, as well as in drug discovery research.

Existing approaches evaluate monolayer permeability directly by measuring the amount of a labeled macromolecular tracer traveling through a cell monolayer grown on a porous filter (transwell permeability assays) or indirectly by measurements of electrical resistance across the cell monolayer under different stimulation conditions. ${ }^{6,7}$ The advantage of the transwell permeability assay is its ability to test the size selectivity of intercellular barriers. Limitations include relatively low sensitivity, considerable time between measurements, low throughput format of assay, absolute requirement of complete coverage of transwell membrane by cell monolayer, fluid convection factor and diffusion characteristics of transwell membrane materials that can mask minor changes in monolayer barrier function of endothelium. ${ }^{8}$

The advantage of electrical resistance measurements across a cell monolayer is the ability of data acquisition in real time

Lung Injury Center, Section of Pulmonary and Critical Medicine, Department of Medicine, University of Chicago, Chicago, Illinois, USA

Correspondence: Dr KG Birukov, MD, PhD, Lung Injury Center, Section of Pulmonary and Critical Medicine, Department of Medicine, University of Chicago, $5841 S$. Maryland Avenue, MC-6026, Chicago, IL 60637, USA.

E-mail: kbirukov@medicine.bsd.uchicago.edu

Received 25 June 2012; revised 12 September 2012; accepted 18 September 2012 
and high sensitivity of the method. This technique, however, requires high-cost equipment, provides indirect means for permeability evaluation and usually uses a limited area of cell monolayer for permeability analysis.

Although each existing method offers particular advantages, none allow for spatial resolution of local changes in permeability representing regional variations of endothelial and epithelial barriers in vivo, as reflected by studies of regional heterogeneity of lung injury in vivo. ${ }^{9}$ Existing assays are also unable to evaluate permeability in endothelial or epithelial monolayer grown on elastic substrates and exposed to cyclic mechanical stretch, a key feature of ventilatorinduced lung injury and pathologies associated with over-distension of other organs. We developed and tested a new concept of measurement and visualization of local permeability in cell monolayer exposed to variety of a chemical and mechanical stimuli.

\section{MATERIALS AND METHODS Reagents}

Chemicals and reagents, including collagen type-I (category No.C3511) and gelatin (category No.G2500) were obtained from Sigma-Aldrich (St Louis, MO) unless noted otherwise. FITC-avidin and Texas-Red phalloidin and all reagents for immunofluorescence staining were obtained from Molecular Probes (Eugene, OR). EZ-Link NHS-LC-LC-Biotin (category No.21343) was purchased from Thermo Scientific (Rockford, IL). 8-Bromo-adenosine- $3^{\prime}, 5^{\prime}$-cyclic monophosphate (8BrcAMP) was purchased from EMD Millipore (Billerica, MA). Non-oxidized 1-palmitoyl-2-arachidonoyl-sn-glycero-3phosphorylcholine (PAPC) was obtained from Avanti Polar Lipids (Alabaster, AL). Oxidized PAPC (OxPAPC) was obtained by exposure of dry lipid to air followed by massspectrometry analysis. ${ }^{10} \mathrm{cMyc}$ antibody was purchased from Santa Cruz Biotechnology (Santa Cruz, CA); VE-cadherin antibody was purchased from Cayman (Ann Arbor, MI). 96-Well polystyrene plastic cell culture-certified plates (Costar 3596) were purchased from Corning (Corning, NY). 6-Well culture plates with stretchable silicon-based membrane (BioFlex amino-treated plates) were purchased from Flexcell International (Hillsborough, NC). Rinzle plastic coverslips (category No.72261-18) were purchased from Electron Microscopy Science (Hatfield, PA).

\section{Human Pulmonary Artery Endothelial Cell Culture}

Human pulmonary artery endothelial cells (HPAEC) were obtained from Lonza (Allendale, NJ) and cultured in cell growth medium (EGM-2, Lonza) containing $10 \%$ fetal bovine serum. Cell cultures were maintained at $37^{\circ} \mathrm{C}$ in a humidified 5\% $\mathrm{CO}_{2}$ incubator and used for experiments at passages 5-8. In experiments with agonist stimulation, 10\% fetal bovine serum growth medium was replaced with $2 \%$ fetal bovine serum growth medium for $2 \mathrm{~h}$ before experiment. Measurements of transendothelial electrical resistance across a confluent EC monolayer were performed using the electrical cell-substrate impedance sensing system ECIS-1600 (Applied Biophysics, Troy, NY). Transient transfections with cMyc-tagged RhoA-V14 or Rho kinase-CAT (6-553) were performed using PolyJet transfection reagent (SignaGen, Rockville, MD) according to the manufacturer's protocol. In brief, HPAEC were seeded on 35-mm diameter cell culture dishes with biotinylated gelatin-treated coverslips at a density of $5 \times 10^{5}$ cells per dish. After $24 \mathrm{~h}$, cells were transfected with $1 \mu \mathrm{g}$ of plasmid DNA per dish (ratio: $1 \mu \mathrm{g}$ of DNA per $3 \mu \mathrm{l}$ of PolyJet). Permeability visualization experiments were performed $24 \mathrm{~h}$ after transfection.

\section{Gelatin/Collagen Biotinylation and Adsorption on Polystyrene Surfaces and Silicon-based Membranes}

Gelatin from porcine skin (G 2500, Sigma, St. Louis, MO) was dissolved in bicarbonate buffer $\left(0.1 \mathrm{~mol} / \mathrm{l} \mathrm{NaHCO}_{3}\right.$, $\mathrm{pH} 8.3$ ) to a final concentration of $10 \mathrm{mg} / \mathrm{ml}$. The mixture was placed on $70^{\circ} \mathrm{C}$ water bath with constant stirring for complete gelatin dissolution. The solution was clarified by low speed centrifugation $(10000 \mathrm{~g}, 5 \mathrm{~min}$, room temperature). EZ-Link NHS-LC-LC-Biotin dissolved in DMSO $(5.7 \mathrm{mg} / \mathrm{ml})$ was added to gelatin solution at $0.57 \mathrm{mg} / \mathrm{ml}$ final concentration. The biotin conjugation to gelatin was performed for $1 \mathrm{~h}$ at room temperature with constant stirring. Biotinylated proteins were stored in aliquots at $-20{ }^{\circ} \mathrm{C}$. For gelatin adsorption, an aliquot of biotinylated gelatin was thawed in $37^{\circ} \mathrm{C}$ water bath for $10 \mathrm{~min}$, diluted with $0.1 \mathrm{~mol} / \mathrm{l}$ bicarbonate buffer, $\mathrm{pH} 8.3$ to a final concentration 0.25 $\mathrm{mg} / \mathrm{ml}$, sterilized by filtering through $0.22 \mu \mathrm{m}$ filter and added to culture plates/wells. For 96-well plate format, $50 \mu \mathrm{l}$ of biotinylated gelatin per well was added; for BioFlex plate, $3 \mathrm{ml}$ per well was added; and for $35 \mathrm{~mm}$ culture dishes, $2 \mathrm{ml}$ per dish was added. The biotinylated gelatin adsorption was performed at $4{ }^{\circ} \mathrm{C}$ overnight. After protein adsorption, plates/ dishes were washed twice with PBS (pH 7.4, $200 \mu$ l per well), and cells were plated at the desired density. If short-term storage was required, plates/dishes were left in PBS and stored at $4{ }^{\circ} \mathrm{C}$ for up to 2 weeks.

\section{Optimization of FITC-Avidin Binding Efficiency to the Biotinylated Gelatin}

Biotinylated gelatin was diluted in PBS and used in the 0.08$10 \mathrm{mg} / \mathrm{ml}$ concentration range. In all, $50 \mu \mathrm{l}$ of solution was added to each well in a 96-well plate and adsorbed overnight at $4{ }^{\circ} \mathrm{C}$. After the adsorbtion step, the plate was washed twice with $200 \mu$ l of PBS $\left(37^{\circ} \mathrm{C}\right)$. Serial dilutions of $2.5 \mathrm{mg} / \mathrm{ml}$ FITC-avidin stock solution were prepared in PBS. In all, $100 \mu \mathrm{l}$ of FITC-avidin solution was added to each well in the 96-well plate and the binding reaction was performed for $1 \mathrm{~min}$ at room temperature. Unbound FITC-avidin was removed by two rounds of plate washing with PBS $(200 \mu \mathrm{l}$ per well). The impact of non-specific binding of FITC-avidin on fluorescence readings of this essay was evaluated in initial tests. Addition of FITC-avidin to 96-well polysterene culture plates coated with unlabeled gelatin or to untreated plates 
followed by FITC-avidin washout or addition of FITC-avidin to uncoated plastic dishes with grown ECs did not increase fluorescence signal above background level (data not shown).

\section{6-Well Assay}

For the permeability assay in the 96-well plates, cells were seeded on biotinylated gelatin-coated plates $\left(3 \times 10^{4}\right.$ cells/ well) and grown for $48-72 \mathrm{~h}$ before testing. FITC-avidin solution was added directly to the culture medium at the final concentration $25 \mu \mathrm{g} / \mathrm{ml}$ for $3 \mathrm{~min}$ before termination of the experiment unless otherwise specified. Unbound FITCavidin was washed out with $200 \mu \mathrm{l}$ PBS, pH $7.4,37^{\circ} \mathrm{C}$ (two cycles, $10 \mathrm{~s}$ each). Finally, $100 \mu \mathrm{l}$ PBS was added in each well, and the fluorescence of matrix-bound FITC-avidin was measured on Victor X5 Multilabel Plate Reader (Perkin Elmer, Waltham, MA) using an excitation wavelength of $485 \mathrm{~nm}$ and emission wavelength of $535 \mathrm{~nm}, 0.1 \mathrm{~s}$.

\section{Permeability Visualization}

Permeability visualization experiments were performed on $18 \times 18 \mathrm{~mm}$ square Rinzle plastic coverslips. The coverslips, placed in $35 \mathrm{~mm}$ culture dishes, were coated with biotinylated gelatin as described above. HPAEC were seeded $(4.5 \times$ $10^{5}$ cells/dish) and grown for $48-72 \mathrm{~h}$ to reach confluence. Cells were transferred to EGM-2 containing 2\% fetal bovine serum $2 \mathrm{~h}$ before agonist stimulation. At the time point of permeability evaluation, FITC-avidin $(25 \mu \mathrm{g} / \mathrm{ml}$ final concentration) was directly added to the culture medium for $3 \mathrm{~min}$ followed by two washing steps ( $3 \mathrm{ml}$ of PBS, $\mathrm{pH} 7.4$, $37^{\circ} \mathrm{C}$ ) and cell fixation with $3.7 \%$ formaldehyde in PBS (10 min, room temperature). Immunofluorescence staining of F-actin, VE-cadherin and tagged, overexpressed proteins was performed as described elsewhere. ${ }^{11}$ Images were acquired using Nikon video imaging system Eclipse TE 300 (Nikon, Tokyo, Japan) equipped with a digital camera (DKC 5000, Sony, Tokyo, Japan); $\times 10$ and $\times 60$ objective lenses were used. Images were processed with Adobe Photoshop 7.0 software (Adobe Systems, San Jose, CA).

\section{Permeability Measurement During Cyclic Stretch}

CS experiments were performed using FX-4000T Flexcell Tension Plus system (Flexcell International, Hillsborough, NC) equipped with $25 \mathrm{~mm}$ BioFlex Loading station, as previously described. ${ }^{11}$ Coating of BioFlex plates with biotinylated gelatin was performed as described above. Cells were seeded at density $5 \times 10^{5}$ cells per well and grown for $48-72 \mathrm{~h}$ to reach confluence. Cells were exposed to high magnitude CS (equibiaxial stretch, 18\% linear elongation, sinusoidal wave, 25 cycles/min) to recapitulate the mechanical stress experienced by the alveolar endothelium during mechanical ventilation at high tidal volume. After $2 \mathrm{~h}$ of CS stimulation, cells were treated with vehicle or thrombin under continuing CS. Control BioFlex plates with static cell culture placed in the same cell culture incubator and processed similarly to the stretch-preconditioned cells were used as control. At the end of the experiment, FITC-avidin (final concentration $25 \mu \mathrm{g} / \mathrm{ml}$ ) was added to the culture medium for $3 \mathrm{~min}$. Unbound FITC-avidin was removed by two-step washing with $3 \mathrm{ml}$ of PBS $\left(37^{\circ} \mathrm{C}\right)$. Elastic bottoms of BioFlex plates with HPAEC were excised with a scalpel and transferred to a polystyrene 6-well cell culture plate. Each membrane was covered with $1 \mathrm{ml}$ of PBS and the fluorescence of membrane-bound FITC-avidin was measured with Victor X5 Multilabel Plate Reader as describe above.

\section{Statistical Analysis}

Results are expressed as mean \pm s.d. Experimental samples were compared with controls by unpaired Student's $t$-test. For multiple-group comparisons, a one-way analysis of variance and Tukey's post hoc multiple-comparison test were used. $P<0.05$ was considered statistically significant. Ten independent experiments were performed to evaluate effects of protective and barrier-disruptive agonists in 96-well assay format; three independent experiments were performed to demonstrate the thrombin time course in 96-well assays; four independent experiments were done for VE-cadherin/FITC visualization; three independent experiments for cMyc-tag/ FITC visualization; and five independent CS-permeability experiments were performed. All measurements were performed in triplicates.

\section{RESULTS \\ The Concept of XPerT Permeability Assay}

To overcome the drawbacks of existing technologies, we utilized the culture plate surface underlying growing cells as a permeability detection surface. In this assay, the permeability is measured as the bulk amount of the fluorescently labeled ligand (ie, FITC-avidin) bound to the surfaceimmobilized high-affinity acceptor underneath the cell monolayer (Figure 1).

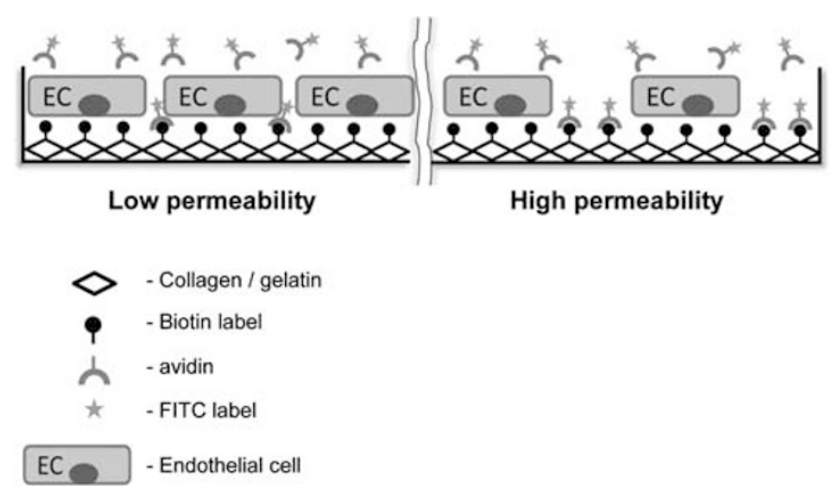

Figure 1 Assay principle. Cells are grown on a surface coated with biotinylated adhesive matrix (ie, gelatin or collagen-I). Permeability is assayed by direct addition of FITC-labeled avidin to the culture medium. FITC-avidin binds biotin moieties conjugated to underlying substrate and accessible through intercellular pores and openings or via transcellular mass transport pathways. The cell monolayer permeability index corresponds to the amount of FITC-avidin bound to biotinylated cell adhesive substrate. 
In the prototype assay, biotinylated gelatin absorbed on a polystyrene or silicon-based surface was used as the detection surface, and FITC-labeled avidin was used as the permeability tracer. This selection was dictated by (1) high-affinity acceptor-ligand interaction $\left(K_{\mathrm{d}} \approx 10^{15}\right)$ in most biological buffers; ${ }^{12}$ (2) flexibility of biotinylation techniques that can be applied to different substrates; and (3) wide range of chemical conjugates of avidin and its bacterial analog streptavidin, which can be used for various modes of detection (ie, multicolor fluorescent or enzymatic-based assay platforms).

\section{Assay Optimization and Testing in Cell Culture}

Titration experiments defined the optimal adsorbtion of biotinylated gelatin to the polysterene multiwell plates that was tested by quantification of FITC-labeled avidin bound to biotinylated gelatin-coated cell-free 96-well plates (Figure 2a). Adsorbtion of biotinylated gelatin reached plateau levels at $0.08 \mathrm{mg} / \mathrm{ml}$. FITC-labeled avidin at a concentration of $25 \mu \mathrm{g} / \mathrm{ml}$ was used in the following studies (Figure 2a). Initial tests showed that binding of soluble FITC-biotin conjugates to avidin immobilized on the plastic surfaces was less efficient and reliable (data not shown). Biotinylated gelatin-coated culture plates were further tested in cell culture experiments.

Because a certain time is required for cell attachment, spreading and formation of a continuous cell monolayer, it is possible that growing cells can degrade the underlying biotinylated substrate and compromise the sensitivity of the assay. These potential effects were tested using HPAEC grown during 24, 72, 96 and $120 \mathrm{~h}$ in multiwell plates coated with biotinylated gelatin $(0.25 \mathrm{mg} / \mathrm{ml})$. The FITC-avidin-binding capacity of cell-preconditioned biotinylated gelatin matrix was evaluated after cell removal with $50 \mathrm{mM}$ EDTA and addition of FITC-avidin at saturating concentration. Cell preconditioning for up to $120 \mathrm{~h}$ showed no effect on the FITC-avidin-binding capacity to the adsorbed biotinylated gelatin (Figure 2b). Similar results were obtained with immobilized biotinylated collagen-I (data not shown). These data indicate high signal stability during cell growth in this assay.

The time window of cell monolayer incubation with FITC-avidin for optimal detection of permeability was further defined. Cells were stimulated with thrombin for $15 \mathrm{~min}$, and FITC-avidin was added for $30 \mathrm{~s}, 1,2,3$ or 5 min before the termination of the experiment and fluorescence measurements. The 3-min incubation with FITC-avidin provided the optimal fluorescence signal, reflecting the cell monolayer barrier state (Figure 2c).

The optimal conditions of cell permeability assays defined in these optimization experiments were used in all the subsequent experiments (immobilization of biotinylated gelatin $-0.25 \mathrm{mg} / \mathrm{ml}, 18 \mathrm{~h},+4{ }^{\circ} \mathrm{C}$; permeability detection by 3 -min addition of $25 \mu \mathrm{g} / \mathrm{ml}$ FITC-avidin. Cells were grown
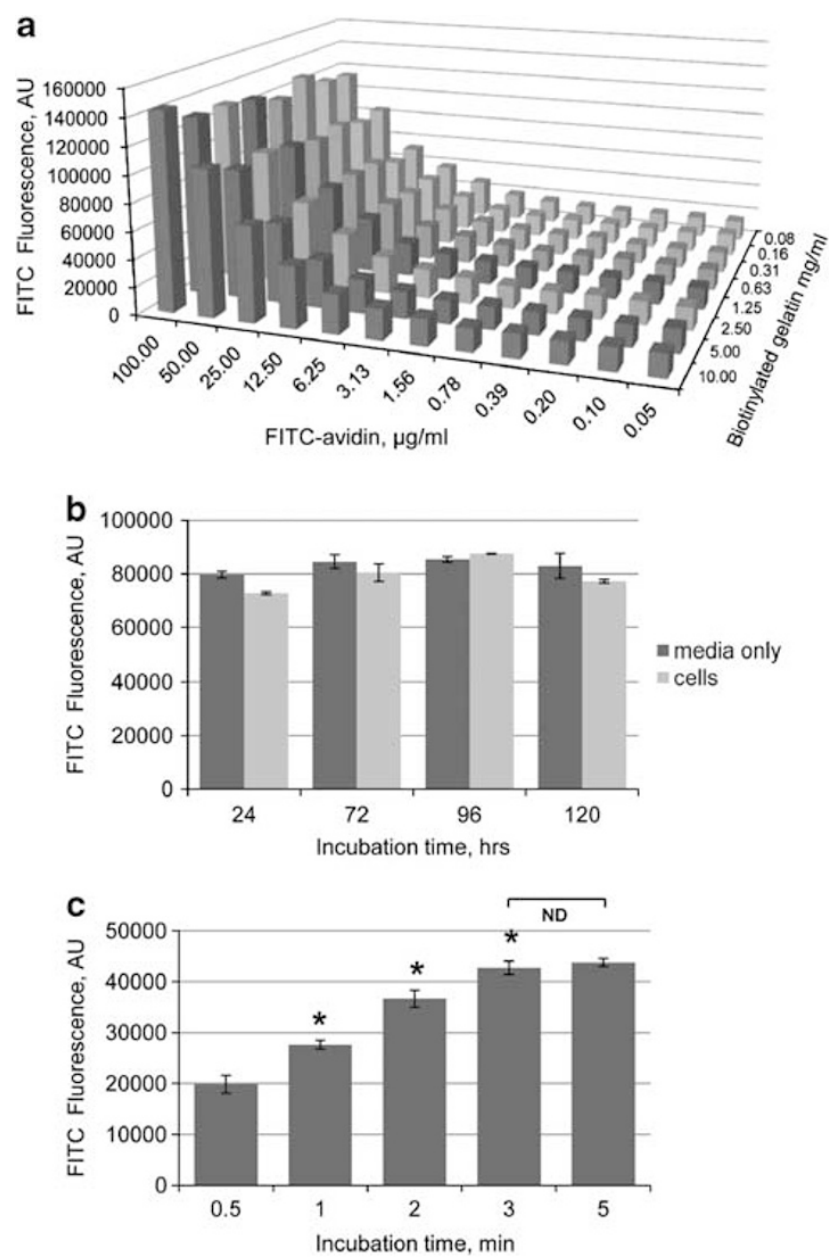

Figure 2 Assay optimization. (a) Titration of FITC-avidin-biotinylated gelatin binding ratio. Gelatin was biotinylated as described in the Materials and Methods section and adsorbed on a 96-well polystyrene plate at the indicated concentrations. FITC-avidin at the $50-0.024 \mu \mathrm{g} / \mathrm{ml}$ concentration range was added to the assay plate (100 $\mu \mathrm{l}$ per well) for $1 \mathrm{~min}$ at room temperature. Unbound FITC-avidin was removed, and FITC fluorescence at the bottom of culture dish was measured. (b) Effect of cell culture on the FITC-avidin-binding capacity of surface-adsorbed biotinylated gelatin. HPAEC were cultured in a 96-well plate coated with biotinylated gelatin $(0.25 \mathrm{mg} / \mathrm{ml}$, overnight incubation) for 24,72 , 96 or $120 \mathrm{~h}$. At the end of cell incubation time, cells were detached by treatment with $50 \mathrm{mM}$ EDTA in PBS $\left(37^{\circ} \mathrm{C}, 10 \mathrm{~min}\right)$, and measurements of FITC-avidin-binding capacity of cell-preconditioned substrate were performed. Plates coated with biotinylated gelatin and similarly processed without HPAEC culturing served as controls. (c) Optimization of FITC-avidin incubation time for detection of thrombin-induced permeability. HPAEC grown in 96-well plates with immobilized biotinylated gelatin $(0.25 \mathrm{mg} / \mathrm{ml})$ were treated with $0.3 \mathrm{U} / \mathrm{ml}$ of thrombin (15 min), and FITC-avidin $(25 \mu \mathrm{g} / \mathrm{ml})$ was added for the indicated time periods. Unbound FITC-avidin was removed, and FITC fluorescence was measured.

on the biotinylated gelatin for $24-48 \mathrm{~h}$ before permeability testing.

\section{Application of XPerT for High Throughput Screening}

An EC monolayer maintains a basal level of permeability, which can be dynamically regulated by pathological and 
physiological stimuli. Adaptation of the XPerT method to a 96-well format allows analysis of time-dependent permeability responses in one assay. Thrombin stimulation was used as an established model of agonist-induced endothelial barrier compromise and led to the time-dependent and reversible increase in substrate-associated FITC fluorescence, reflecting increased permeability (Figure 3a).

The high throughput format of this assay allows for rapid simultaneous measurements of cell monolayer permeability changes induced by a variety of bioactive molecules and their combinations. The capacity of this method to simultaneously analyze both the barrier-disruptive and barrier-enhancing cell responses to various agonists and their combination was further tested.

Enhancement of endothelial monolayer barrier properties by oxidized phospholipids (OxPAPC) or elevation of intracellular cyclic AMP levels using 8Br-cAMP and forskolin has been previously demonstrated using transwell assays and transendothelial electrical resistance measurements. ${ }^{10,13}$ We compared results of human EC permeability testing using TER measurements and the XPerT assay in 96-well format.

OxPAPC, 8Br-cAMP and forskolin significantly decreased the fluorescence signal in the XPerT assay, indicating decreased monolayer permeability, whiereas thrombin markedly increased permeability. Cell pretreatment with OxPAPC, 8Br-cAMP and forskolin attenuated thrombininduced permeability as detected by a decreased fluorescence signal (Figure $3 \mathrm{~b}$ ). These results strongly correlate with independent analysis of permeability responses performed using measurements of transendothelial electrical resistance (Figure 3c).

\section{XPerT Application for Permeability Visualization}

To the best of our knowledge, none of the existing techniques allow for precise and reliable visualization of high permeability regions in a live cell monolayer. Such tools are critically important for understanding the regulation of local permeability and cell junction integrity of vascular endothelium, lung alveolocytes or intestinal epithelium, as a few examples.

Local permeability changes induced by thrombin and forskolin were visualized in cells grown on biotinylated gelatin plastic coverslips. The pattern of FITC-avidin binding to the biotinylated gelatin underlying the cell monolayer was examined under a microscope (Figure 4a). The structural integrity of cell-cell junctions was evaluated in control and agonist-stimulated cell monolayer after FITC-avidin incubation by immunostaining of the adherens junction protein VE-cadherin. Low magnification images demonstrate minimal FITC fluorescence accumulation at the cell-cell junction areas of control cell monolayer, further barrier preservation in forskolin-treated cells and a dramatic increase in FITC fluorescence in the intercellular gaps upon thrombin treatment (Figure 4a). Overlay of FITC and VE-cadherin staining demonstrates reciprocal relations between
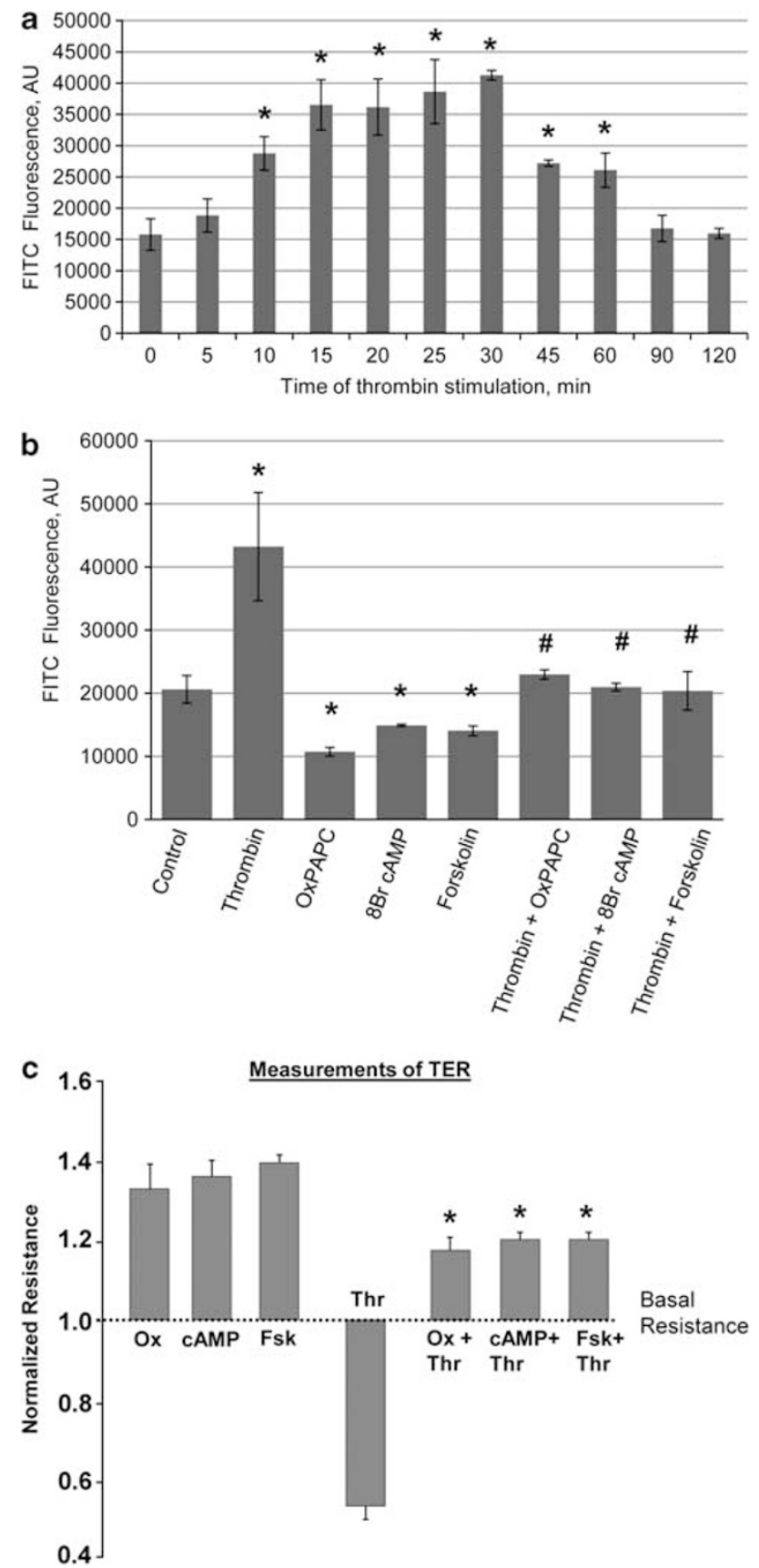

Figure 3 Fluorimetric evaluation of monolayer permeability in 96-well plates. (a) HPAEC were treated with $0.3 \mathrm{U} / \mathrm{ml}$ of thrombin for indicated time periods followed by permeability analysis using measurements of FITC fluorescence. ${ }^{*} P<0.05$ vs control. (b) Cells were pretreated with vehicle, thrombin $(0.3 \mathrm{U} / \mathrm{ml})$ for $15 \mathrm{~min}$, or OxPAPC $(15 \mu \mathrm{g} / \mathrm{ml})$, 8Br-cAMP $(500 \mu \mathrm{M})$ or forskolin $(2 \mu \mathrm{M})$ for $30 \mathrm{~min}$. Alternatively, cells preincubated for $30 \mathrm{~min}$ with OxPAPC, 8Br-cAMP or forskolin were stimulated with thrombin $(0.3 \mathrm{U} / \mathrm{ml}, 15 \mathrm{~min}) .{ }^{*} P<0.05$ vs control; ${ }^{\#} P<0.05$ vs thrombin alone. (c) Measurements of transendothelial electrical resistance across confluent HPAEC were performed as described in the Materials and Methods section. Bar graph represents normalized cell monolayer resistance measured in the same conditions as panel $\mathbf{b}$. ${ }^{*} P<0.05$ vs thrombin alone. FITC-avidin binding correlates with measurements of transendothelial resistance. 


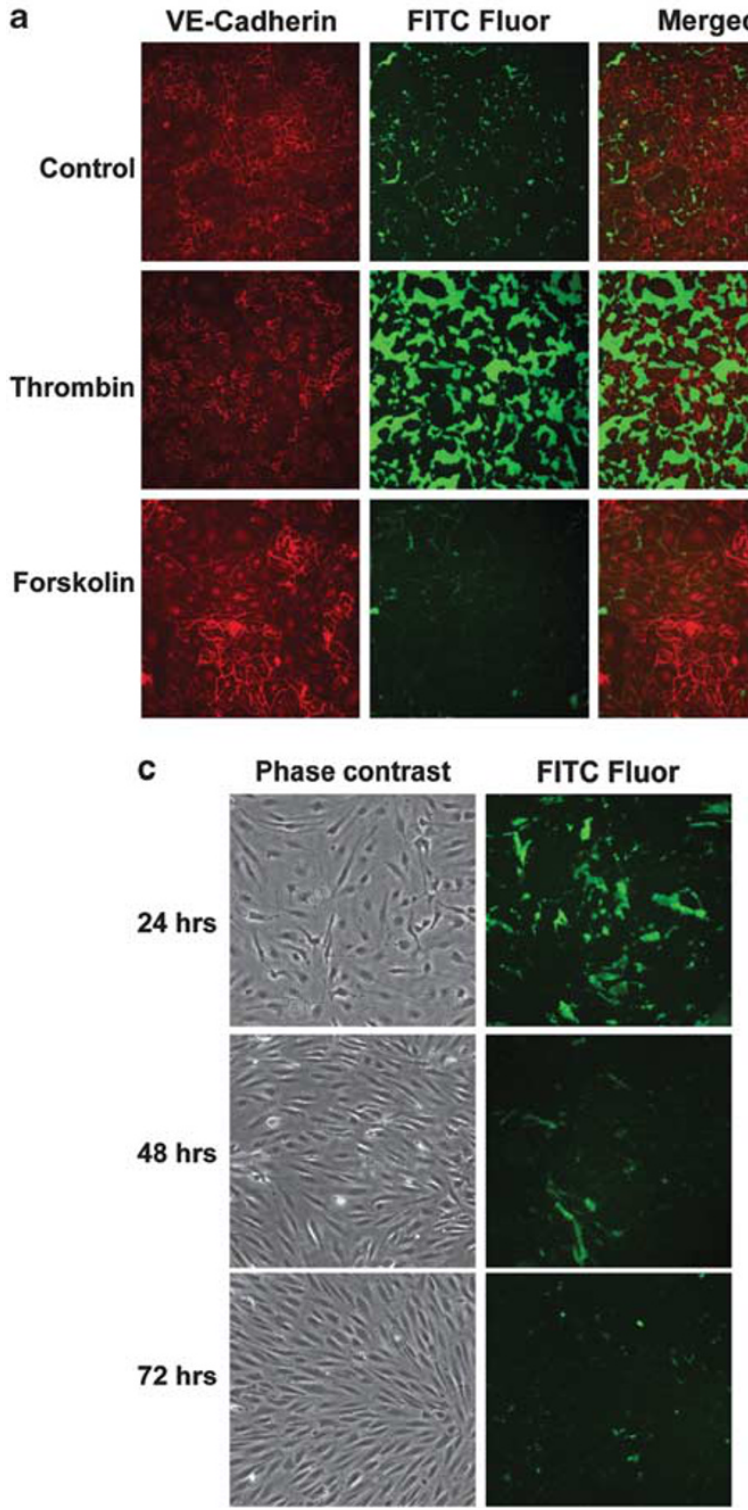

b
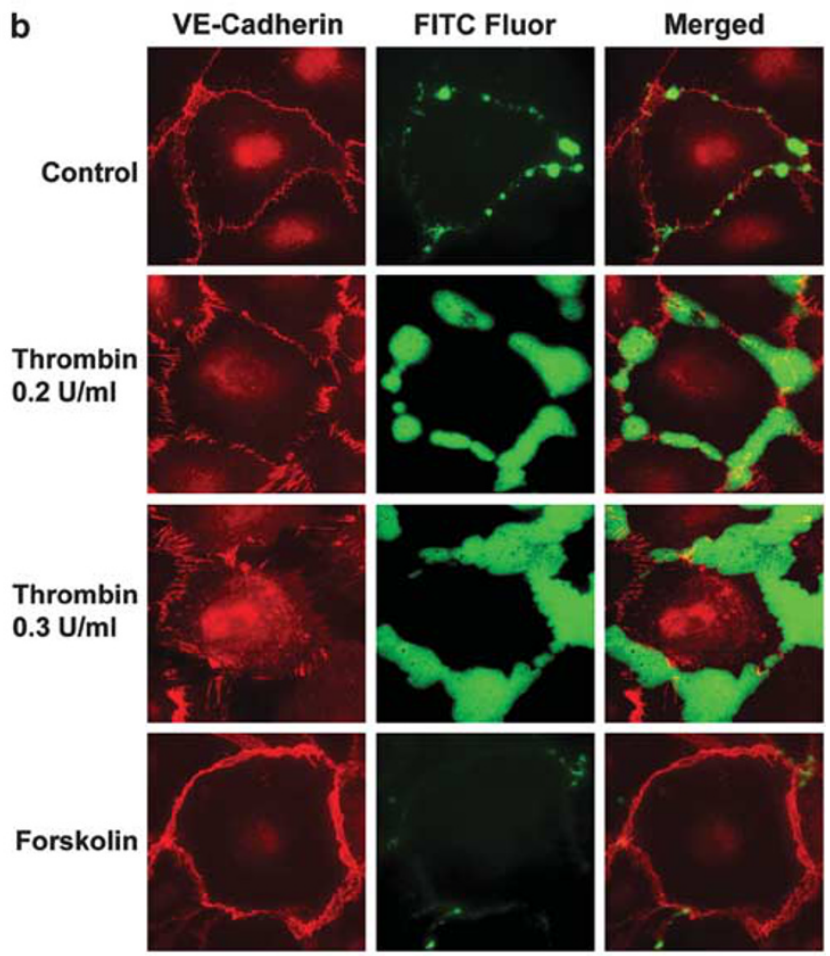

Figure 4 Visualization of agonist-induced permeability in endothelial monolayers. (a, b) HPAEC were plated on coverslips coated with biotinylated gelatin and grown to confluence. HPAEC were treated with thrombin $(0.2$ or $0.3 \mathrm{U} / \mathrm{ml}, 15 \mathrm{~min})$ or forskolin $(2 \mu \mathrm{M}, 30 \mathrm{~min})$. At the end of the experiment, FITC-avidin was added for $3 \mathrm{~min}$, cells were fixed and subjected to immunfluorescence staining for VE-cadherin (red) to visualize cell-cell contacts. Green fluorescence depicts areas permeable for FITC-labeled avidin. Images were taken using (a) $\times 10$ and (b) $\times 60$ objectives. (c) Intercellular gap closure during HPAEC growth was monitored by decreased number of FITC-positive intercellular spots after 24,48 and $72 \mathrm{~h}$ of cell culture.

VE-cadherin peripheral localization and increased local permeability of FITC-avidin.

This novel assay also allows for high-resolution visualization of permeability sites in the cell monolayer and their relation to local changes in cytoskeletal or cell-adhesion structure. Basal permeability of non-stimulated pulmonary endothelium can be seen as a thin ring of FITC-avidin fluorescence surrounding cells with a few larger pores, indicating increased local permeability (Figure $4 \mathrm{~b}$, upper row). Thrombin treatment caused a dose-dependent, non-uniform increase in intercellular permeability reflected by enlarged areas of intercellular fluorescence (Figure $4 \mathrm{~b}$, middle rows).
By contrast, forskolin diminished basal permeability levels. These changes were associated with enlargement of cell-cell junction complexes detected by VE-cadherin staining (Figure 4b, lower row).

The stability of biotinylated gelatin or collagen matrices immobilized on culture dishes (characterized in Figure $2 \mathrm{~b}$ ) also allows for evaluation of monolayer permeability over the course of several days. Therefore, the XPerT assay can be used to monitor the confluency state of the cell monolayer in chronic experiments (Figure 4c).

A major limitation of existing molecular approaches to dissect pathways regulating cell permeability is the inability 
to visualize local permeability rendered by transfected cells within the intact monolayer. The suitability of the XPerT method to address this problem was tested in cells transiently transfected with constitutively activated Rho kinase (Rho kinase-CAT) or the activated RhoA mutant (RhoA-V14). These activated proteins increased endothelial permeability. ${ }^{14,15}$ Increased permeability was observed in the area surrounding cells expressing activated Rho kinase (Figure 5a) and activated RhoA (Figure 5b). No changes in local permeability can be seen in non-transfected cells (Figures 5a and $b$, top panels) or cells transfected with control vector (data not shown). In comparison, cytoskeletal staining of transfected and non-transfected cells in monolayer cultures did not provide reliable visualization of the areas with increased permeability (Figures $5 \mathrm{a}$ and $\mathrm{b}$, bottom panels).

\section{XPerT: Permeability Measurements in Cell Monolayer on Elastic Substrates Under Mechanical Stimulation}

The physical forces are important regulators of endothelial and epithelial barrier function ${ }^{16-19}$ and approaches to measure permeability in cells exposed to mechanical stimulation are in high demand. Until now, such techniques were unavailable. To overcome this methodological constraint, we tested the potential application of the XPerT assay to the flexible-bottomed culture plates that can be used for exposure of various cells to CS. The biotinylated gelatin coating protocol was adapted for silicone-based stretch plates, as the first example of an elastic substrate.

The permeability of the endothelial monolayer under static conditions and upon exposure to pathologically relevant CS magnitude (18\% CS $)^{20}$ was further evaluated by measurement of FITC fluorescence intensity of the bottom membranes excised from the stretch plates. Two-hour exposure of cells to $18 \%$ CS induces cytoskeletal reorientation, without visible alterations in monolayer integrity (Figure 6a). However, application of the CS-adapted XPerT assay allows, for the first time, the direct detection of increased leakiness in endothelial monolayer exposed to pathological CS (Figure 6b). Thrombin treatment of static cells and cells exposed to continuous CS further increased a

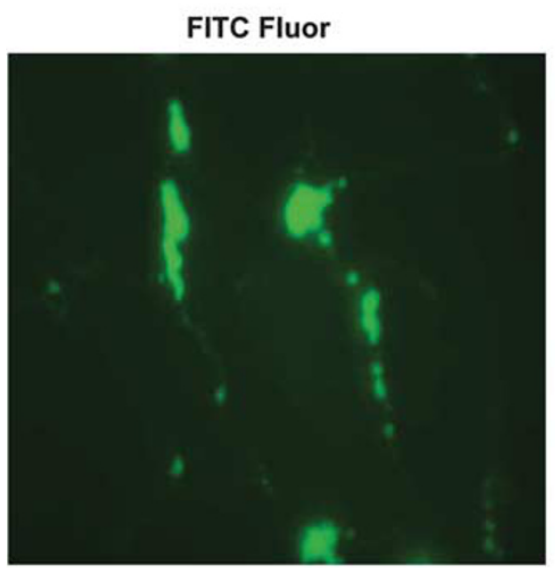

F-Actin

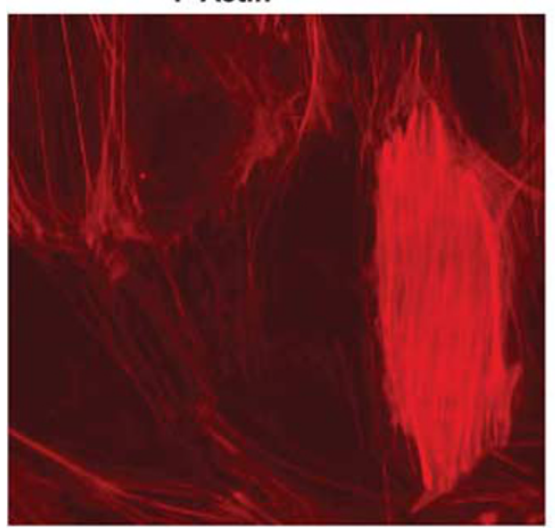

TF Rho Kinase-CAT

cMyc-Tag

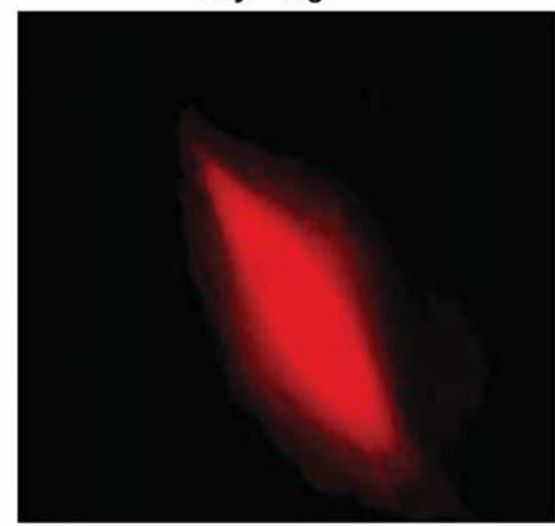

cMyc-Tag

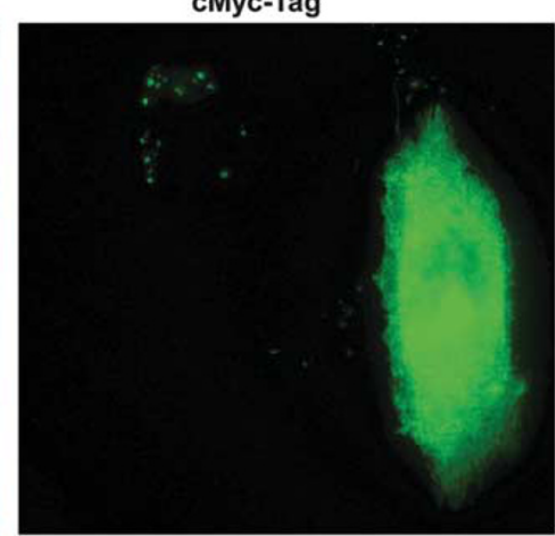

Merged

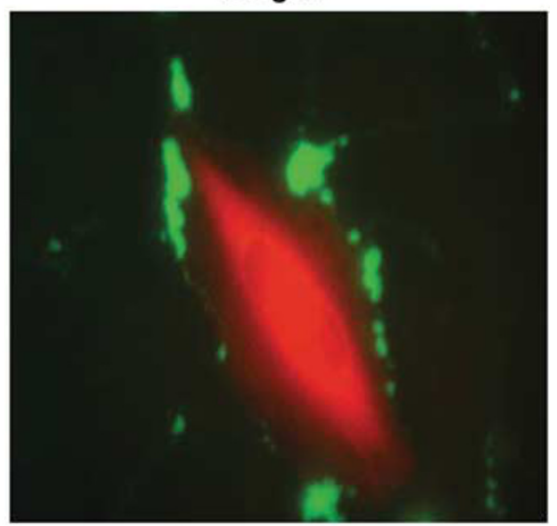

Merged

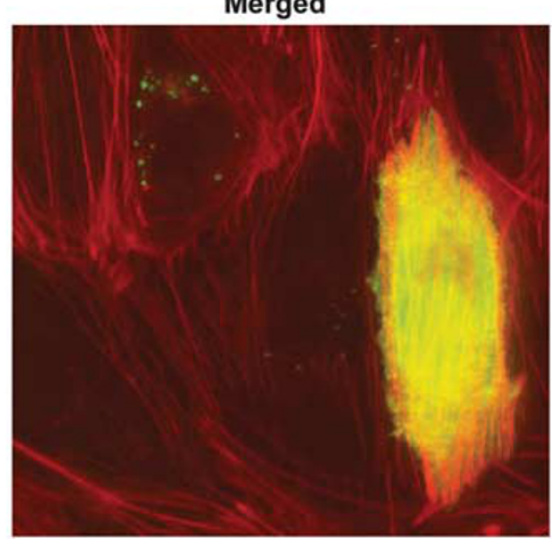

Figure 5 Visualization of local cell permeability. HPAEC seeded on biotinylated gelatin-coated coverslips were transfected with (a) cMyc-tagged activated Rho kinase-CA or (b) CMyc-tagged RhoA-V14 mutant followed by incubation with FITC-avidin and immunofluorescence staining for CMyc tag (red). Green fluorescence depicts areas permeable to FITC-labeled avidin. Merged images show areas of increased local permeability surrounding cells expressing activated RhoA. Lower panels depict staining of F-actin with Texas-Red phalloidin (red) and cMyc tag (green) performed in parallel experiments. Cytoskeletal staining detects large paracellular gaps but does not distinguish precise spots of increased permeability. 
b

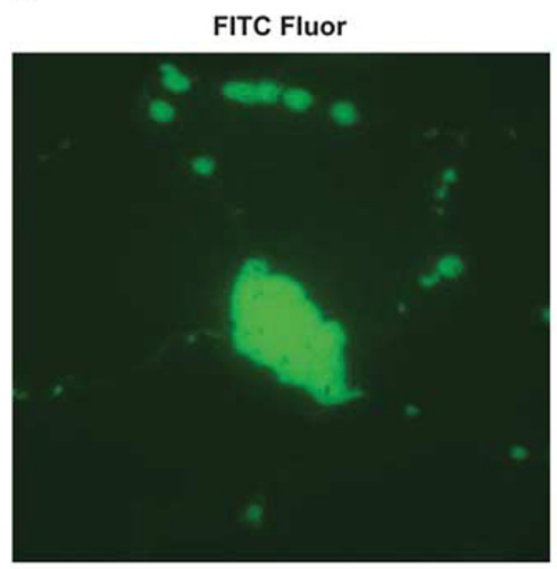

F-Actin

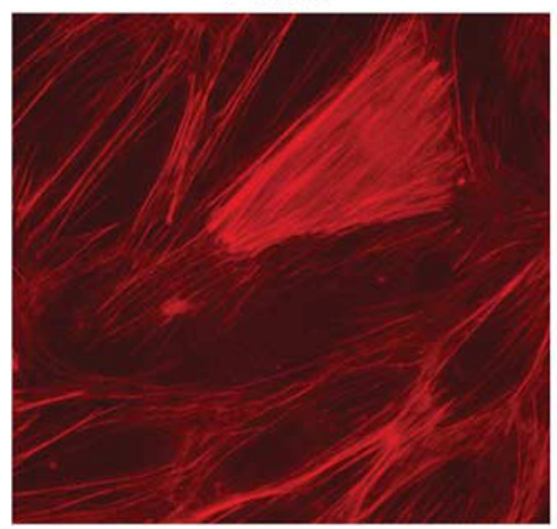

TF RhoA-V14

cMyc-Tag

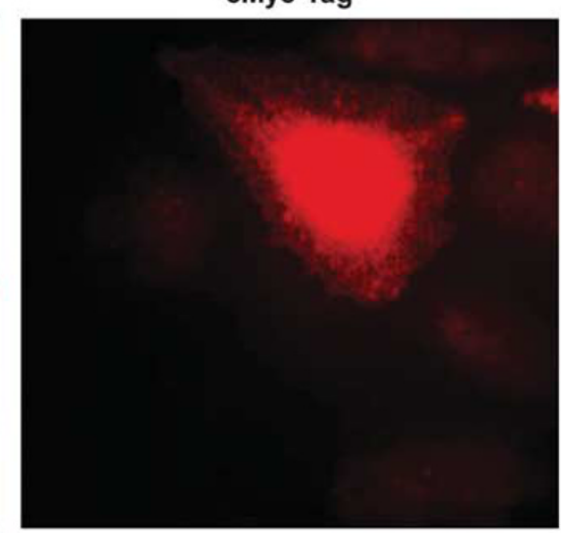

cMyc-Tag

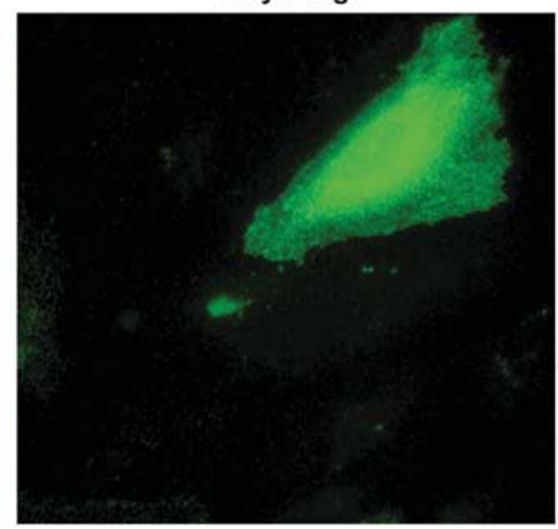

Merged

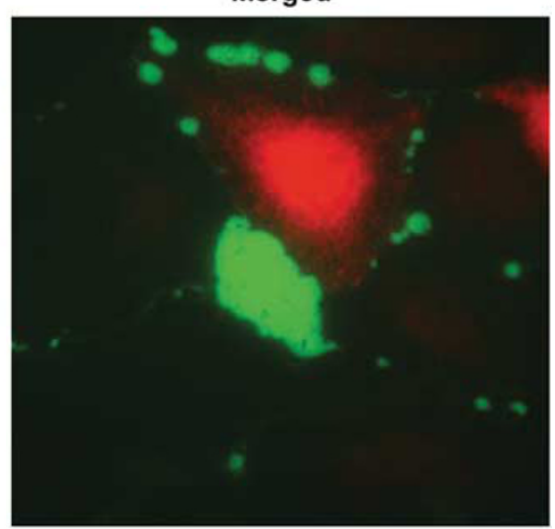

Merged

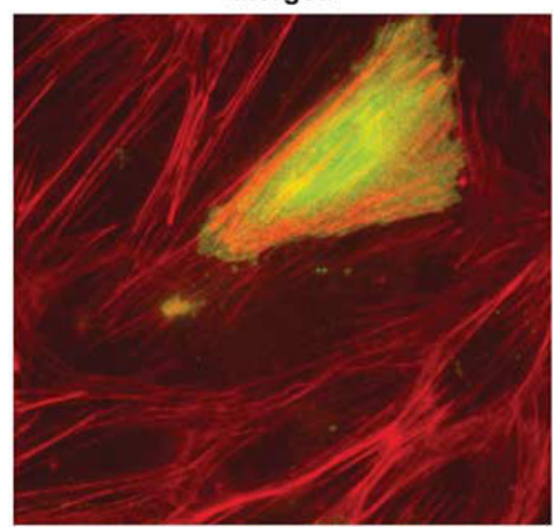

Figure 5 (Conintued)

monolayer permeability. These experiments illustrate a significant advancement in existing approaches for evaluation of monolayer barrier properties in static and a mechanically active environment that have been previously possible only by means of morphometric analysis of visible cell-denuded areas. ${ }^{20,21}$

\section{DISCUSSION}

We report a novel concept for a cell permeability assay based on high-affinity ligand-acceptor interactions and describe several applications of this methodology for analysis of novel aspects of monolayer permeability. Monolayer permeability can be evaluated by this method in bulk assays using quantification of fluorescence by a microplate reader or may engage fluorescence microscopy and image analysis to determine spatial distribution of permeability sites in a cell monolayer.

Although the role of specific cytoskeletal patterns of remodeling and rearrangements in cell junction complexes have been linked to alterations of EC monolayer permeability, ${ }^{22}$ direct comparison of cytoskeletal remodeling by cell monolayer with areas of increased leakiness has not been previously made possible. Permeability maps obtained using the XPerT approach from microscopic images of cells under physiological and pathological conditions may be further used for quantitative permeability analysis. This study demonstrates, for the first time, the reciprocal patterns of local permeability detected by the XPerT assay and heterogeneous distribution of VE-cadherin, the protein having a major role in the maintenance of the endothelial barrier. ${ }^{23}$ Another important feature of this method is its ability to evaluate local permeability at a single-cell level. This feature is critical for molecular analysis of genes and proteins regulating cell-cell interactions under pathological conditions.

Utilization of the XPert approach for permeability analysis in EC monolayer grown on stretchable substrates has been further tested in this study. This approach allowed, for the first time, the assessment of basal permeability of endothelial monolayer under continuous pathological CS, which cannot be achieved using other existing approaches. Integration of mechanotransduction studies linking mechanochemical signaling pathways with analysis of stretch-induced monolayer permeability changes will bring new dimensions in this area of research and will bridge in vitro studies and animal models of disease.

The concept and its biological applications described in this study suggest the potential for further technological developments based on this principle. First, substrate-coating techniques can be adapted to glass surfaces, which will 
a

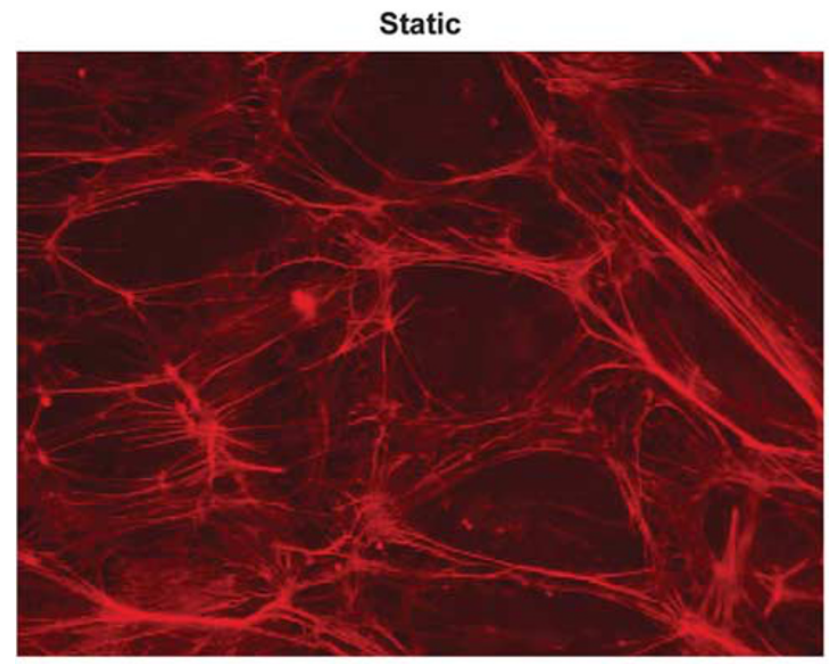

F-Actin

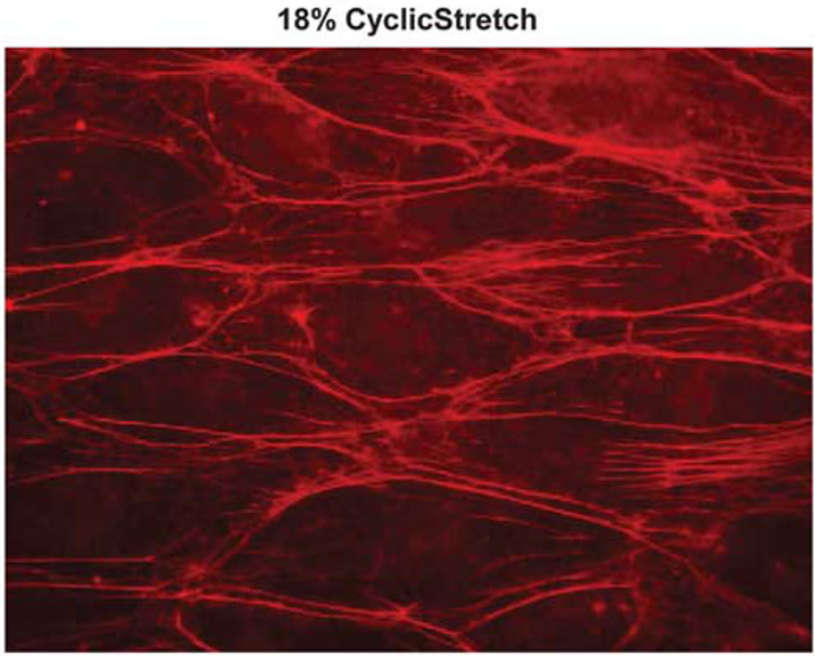

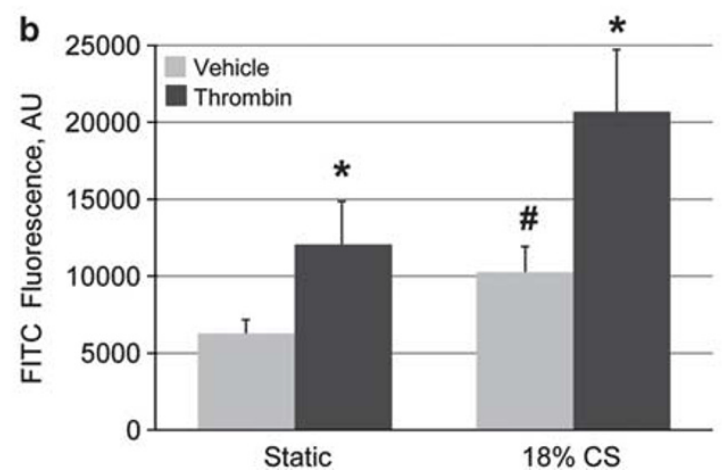

Figure 6 Measurements of endothelial monolayer permeability under cyclic stretch. (a) Confluent HPAEC grown on Flexcell plates were exposed to $18 \%$ cyclic stretch for $2 \mathrm{~h}$ or left static. F-actin arrangement in static and CS-exposed cells was visualized using Texas-Red phalloidin. (b) Flexcell plates coated with biotinylated gelatin were seeded with HPAEC and exposed to $18 \%$ cyclic stretch for $2 \mathrm{~h}$ or left static. After $2 \mathrm{~h}$ of stretch preconditioning, cells were stimulated with thrombin $(0.3 \mathrm{U} / \mathrm{ml}, 15 \mathrm{~min})$ or vehicle. FITC-avidin was added as described above, and FITC fluorescence of silicon bottom membranes was measured using Victor $X 5$ plate reader. ${ }^{*} P<0.05$ vs vehicle, ${ }^{\#} P<0.05$ vs static.

provide superior image quality, or to other substrates currently used in tissue engineering and cell biology. The XPerT platform can be also incorporated in the microfluidics devices. Second, the versatility of the XPerT platform can be further expanded by testing a variety of other high-affinity binary compounds. Examples include high-affinity Fc fragment-protein A or GST-glutathione interactions. Alternatively, utilization of substrate-enzyme pairs may significantly increase the sensitivity of such assays and allow permeability analysis in real time using fluorometric or spectrophotometric detection methods.

In conclusion, the novel approach for visualization and quantization of local permeability at the subcellular level in cell cultures exposed to static or pathological CS conditions introduces a new dimension into studies of pathologies associated with disruption of monolayer integrity and increased permeability under dynamic mechanochemical microenvironments. ${ }^{20,24-26}$ We provided, for the first time, the direct evidence that local activation of Rho signaling caused by overexpression of constitutively active Rho GTPase or Rho kinase in pulmonary EC caused a local increase in permeability. We demonstrated direct effects of pathological CS on EC monolayer permeability and observed synergistic effects of pathological CS and thrombin on EC permeability increase that previously was only performed indirectly, via analysis of visible gap formation. These experiments reproduce the pathological mechanochemical environment experienced by pulmonary vascular endothelium in clinical settings of ventilator-induced lung injury/ARDS. ${ }^{27-29}$ The results of this study strongly suggest that the proposed approach will help overcome existing limitations faced by investigators studying molecular mechanisms of mechanotransduction, barrier regulation by pathological mechanical forces and preservation of monolayer integrity in health and disease.

\section{ACKNOWLEDGEMENTS}

We thank Kozo Kaibuchi (Nagoya University, Japan) for his generous gift of RhoA and Rho kinase plasmids and Katherine Higginbotham for proofreading the manuscript. This work was supported by HL087823, HL076259 (to KGB) and HL107920 (to AAB). 


\section{DISCLOSURE/CONFLICT OF INTEREST}

The authors declare no conflict of interest.

1. Hirase T, Node K. Endothelial dysfunction as a cellular mechanism for vascular failure. Am J Physiol Heart Circ Physiol 2012;302:H499-H505.

2. Le Guelte A, Dwyer J, Gavard J. Jumping the barrier: VE-cadherin VEGF and other angiogenic modifiers in cancer. Biol Cell 2011;103: 593-605.

3. Fraser PA. The role of free radical generation in increasing cerebrovascular permeability. Free Radic Biol Med 2011;51:967-977.

4. Fishel RS, Are C, Barbul A. Vessel injury and capillary leak. Crit Care Med 2003;31(8 Suppl):S502-S511.

5. Ware LB, Matthay MA. Clinical practice. Acute pulmonary edema. N Engl J Med 2005;353:2788-2796.

6. Balda MS, Whitney JA, Flores C, et al. Functional dissociation of paracellular permeability and transepithelial electrical resistance and disruption of the apical-basolateral intramembrane diffusion barrier by expression of a mutant tight junction membrane protein. J Cell Biol 1996;134:1031-1049.

7. Giaever I, Keese CR. Monitoring fibroblast behavior in tissue culture with an applied electric field. Proc Natl Acad Sci USA 1984;81: 3761-3764.

8. Lo CM, Keese CR, Giaever I. Cell-substrate contact: another factor may influence transepithelial electrical resistance of cell layers cultured on permeable filters. Exp Cell Res 1999;250:576-580.

9. McVerry BJ, Peng X, Hassoun PM, et al. Sphingosine 1-phosphate reduces vascular leak in murine and canine models of acute lung injury. Am J Respir Crit Care Med 2004;170:987-993.

10. Birukov KG, Bochkov VN, Birukova AA, et al. Epoxycyclopentenonecontaining oxidized phospholipids restore endothelial barrier function via Cdc42 and Rac. Circ Res 2004;95:892-901.

11. Birukova AA, Fu P, Xing J, et al. Mechanotransduction by GEF-H1 as a novel mechanism of ventilator-induced vascular endothelial permeability. Am J Physiol Lung Cell Mol Physiol 2010;298:L837-L848.

12. Green NM. Avidin. 3. The Nature of the Biotin-Binding Site. Biochem J 1963;89:599-609.

13. Fukuhara S, Sakurai A, Sano H, et al. Cyclic AMP potentiates vascular endothelial cadherin-mediated cell-cell contact to enhance endothelial barrier function through an Epac-Rap1 signaling pathway. Mol Cell Biol 2005;25:136-146.

14. van Nieuw Amerongen GP, van Delft S, Vermeer MA, et al. Activation of RhoA by thrombin in endothelial hyperpermeability: role of Rho kinase and protein tyrosine kinases. Circ Res 2000;87:335-340.
15. Wojciak-Stothard B, Ridley AJ. Rho GTPases and the regulation of endothelial permeability. Vascul Pharmacol 2002;39:187-199.

16. Birukov KG. Small GTPases in mechanosensitive regulation of endothelial barrier. Microvasc Res 2009;77:46-52.

17. Tzima E, Irani-Tehrani M, Kiosses WB, et al. A mechanosensory complex that mediates the endothelial cell response to fluid shear stress. Nature 2005;437:426-431.

18. Sidhaye VK, Schweitzer KS, Caterina MJ, et al. Shear stress regulates aquaporin-5 and airway epithelial barrier function. Proc Natl Acad Sci USA 2008; 105:3345-3350.

19. Cohen TS, Cavanaugh KJ, Margulies SS. Frequency and peak stretch magnitude affect alveolar epithelial permeability. Eur Respir J 2008;32:854-861.

20. Birukova AA, Chatchavalvanich $S$, Rios $A$, et al. Differential regulation of pulmonary endothelial monolayer integrity by varying degrees of cyclic stretch. Am J Pathol 2006;168:1749-1761.

21. Crosby LM, Luellen C, Zhang $Z$, et al. Balance of life and death in alveolar epithelial type II cells: proliferation, apoptosis, and the effects of cyclic stretch on wound healing. Am J Physiol Lung Cell Mol Physiol 2011;301:L536-L546.

22. Mehta $D$, Malik $A B$. Signaling mechanisms regulating endothelial permeability. Physiol Rev 2006;86:279-367.

23. Dejana $E$, Orsenigo F, Lampugnani MG. The role of adherens junctions and VE-cadherin in the control of vascular permeability. J Cell Sci 2008:121(Part 13):2115-2122.

24. Cavanaugh Jr KJ, Margulies SS. Measurement of stretch-induced loss of alveolar epithelial barrier integrity with a novel in vitro method. Am J Physiol Cell Physiol 2002;283:C1801-C1808.

25. Waters CM, Savla U. Keratinocyte growth factor accelerates wound closure in airway epithelium during cyclic mechanical strain. J Cell Physiol 1999;181:424-432.

26. Makena PS, Luellen CL, Balazs $\mathrm{L}$, et al. Preexposure to hyperoxia causes increased lung injury and epithelial apoptosis in mice ventilated with high tidal volumes. Am J Physiol Lung Cell Mol Physio 2010;299:L711-L719.

27. Maniatis NA, Kotanidou A, Catravas JD, et al. Endothelial pathomechanisms in acute lung injury. Vascul Pharmacol 2008:49:119-133.

28. Lionetti V, Recchia FA, Ranieri VM. Overview of ventilator-induced lung injury mechanisms. Curr Opin Crit Care 2005;11:82-86.

29. Matthay MA, Zimmerman GA, Esmon C, et al. Future research directions in acute lung injury: summary of a National Heart, Lung, and Blood Institute working group. Am J Respir Crit Care Med 2003;167:1027-1035. 